ARTICLE

\title{
Live-cell protein labelling with nanometre precision by cell squeezing
}

\author{
Alina Kollmannsperger ${ }^{1}$, Armon Sharei ${ }^{2}$, Anika Raulf ${ }^{3}$, Mike Heilemann $^{3}$, Robert Langer ${ }^{2}$, Klavs F. Jensen ${ }^{2}$, \\ Ralph Wieneke ${ }^{1} \&$ Robert Tampé ${ }^{1,4}$
}

Live-cell labelling techniques to visualize proteins with minimal disturbance are important; however, the currently available methods are limited in their labelling efficiency, specificity and cell permeability. We describe high-throughput protein labelling facilitated by minimalistic probes delivered to mammalian cells by microfluidic cell squeezing. High-affinity and target-specific tracing of proteins in various subcellular compartments is demonstrated, culminating in photoinduced labelling within live cells. Both the fine-tuned delivery of subnanomolar concentrations and the minimal size of the probe allow for live-cell super-resolution imaging with very low background and nanometre precision. This method is fast in probe delivery ( $\sim 1,000,000$ cells per second), versatile across cell types and can be readily transferred to a multitude of proteins. Moreover, the technique succeeds in combination with well-established methods to gain multiplexed labelling and has demonstrated potential to precisely trace target proteins, in live mammalian cells, by super-resolution microscopy.

\footnotetext{
${ }^{1}$ Institute of Biochemistry, Biocenter, Goethe-University Frankfurt, Max-von-Laue Strasse 9, 60438 Frankfurt/Main, Germany. ${ }^{2}$ Department of Chemical Engineering, David H. Koch Institute for Integrative Cancer Research, Massachusetts Institute of Technology (MIT), 500 Main Street, Building 76-661, Cambridge, Massachusetts 02139, USA. ${ }^{3}$ Institute of Physical and Theoretical Chemistry, Goethe-University Frankfurt, Max-von-Laue Strasse 7 , 60438 Frankfurt/Main, Germany. ${ }^{4}$ Cluster of Excellence-Macromolecular Complexes, Goethe-University Frankfurt, Max-von-Laue Strasse 9, 60438 Frankfurt/Main, Germany. Correspondence and requests for materials should be addressed to R.W. (email: wieneke@em.uni-frankfurt.de) or to R.T. (email: tampe@em.uni-frankfurt.de).
} 
$\mathrm{D}$ irect observation of intracellular processes has the potential to yield insight into fundamental biological pathways and disease mechanisms. Several techniques have been developed to enable high-resolution imaging of live cells; yet, the limited ability to trace intracellular components has hindered progress. Hence, two of the persistent challenges are probe design and cellular delivery with minimal toxicity, pivotal for advances in live-cell imaging technologies. Here we describe an efficient approach to tag and image intracellular components in live mammalian cells. Using the microfluidic cell squeezing platform to deliver small fluorescent tris- $\mathrm{N}$-nitrilotriacetic acid (trisNTA) probes $(\sim 1 \mathrm{kDa})$, we demonstrate highly efficient, minimally disruptive, light-triggered tracing of native proteins and the subsequent super-resolution imaging of live-cell phenomena.

Live-cell microscopy contributed significant knowledge of dynamic processes such as protein trafficking and single-molecule localization-based imaging techniques visualize proteins with highresolution information $(\leq 50 \mathrm{~nm})^{1,2}$. All fluorescent imaging techniques require protocols to introduce the label with the need to minimize its influence on the system. Fluorescent proteins, selflabelling tags ${ }^{3-6}$ or labelling by enzymatic methods 7,8 can interfere with protein function, assembly or dynamics. Bulky fusion proteins $(>20 \mathrm{kDa})$ entail the risk of steric hindrance and functional perturbations, whereas smaller tags (for example, tetracysteine tag) deal with unspecific interactions or require additional experimental steps $^{8}$ and optimized flanking sequences for each protein target ${ }^{9}$. Although synthetic fluorophores have enhanced photostability, quantum yield, spectral range and localization precision, it is difficult to introduce such probes to the cytosolic environment using existing delivery technologies. On the one hand, current transduction strategies such as delivery by cell-penetrating peptides (CPPs), electroporation and so on are suboptimal, suffering from poor and endosomal uptake, rapid degradation by extracellular and endosomal proteases, low in vivo efficiency or elaborated chemical synthesis. On the other hand, antibody-based labelling approaches, for example, are limited to chemically arrested (fixed) cells and the availability of specific antibodies for a protein target. Owing to the described limitations of existing labelling and transduction technologies, there is a persistent demand for techniques enabling high-throughput in-cell labelling by minimal tags that are conductive to high-resolution and super-resolution microscopy.

Here we demonstrate robust in-cell targeting of native proteins using a labelled multivalent chelator head tris $\mathrm{NTA}^{10}$ and a genetically encoded oligohistidine sequence (Fig. 1a). trisNTA site specifically recognizes $\mathrm{His}_{6-10}$-tagged proteins in the (sub)nanomolar range ( $K_{\mathrm{d}}$ of $\left.0.1-10 \mathrm{nM}\right)$ even in the crowded cellular environment ${ }^{11}$. The minimal size of the tag and the molecular probe allows direct targeting with nanometre precision at subnanomolar concentrations as required for single-molecule localization-based imaging techniques ${ }^{1,2,12,13}$ with no impact on intracellular trafficking or demand for additional cofactors affecting endogenous processes. We simplified efficient transfer of the trisNTA probe into living cells by cell squeezing ${ }^{14}$, combining precisely controlled cytosolic delivery with high specificity and low cytotoxicity. Briefly, transient cell permeabilization is achieved by rapid viscoelastic deformation of cells as they pass through micrometre-scale constrictions. This facilitates fast uptake of probes into the cytosol before cell-intrinsic repair mechanisms kick in ${ }^{15}$.

\section{Results}

High-affinity protein labelling at subnanomolar concentration. We first investigated the specificity of the tris NTA/His tag targeting in chemically arrested cells. To evaluate precise localization, different proteins resident at distinct subcellular compartments were selected: (i) the transporter associated with antigen processing (TAP) in the membrane of the endoplasmic reticulum ${ }^{16}$; (ii) histone $2 \mathrm{~B}$ (H2B) in the nucleus; and (iii) Lamin $\mathrm{A}$ at the nuclear envelope. All proteins of interest (POIs) were fused to a $\mathrm{His}_{10}$ tag and a fluorescent protein (TAP $1^{\text {mVenus-Hislo, }}$ $\mathrm{H}_{2} \mathrm{~B}^{\mathrm{mV} V e n u s-\mathrm{His} 10}$ and His10-mEGFP Lamin A) for specific targeting and co-localization studies, respectively. For sensitive detection, trisNTA was covalently coupled to different fluorescent dyes $\left(\right.$ tris $\mathrm{NTA}^{\mathrm{f}}, \mathrm{f}=$ Alexa488, ATTO565, ATTO647N, Alexa647 and ATTO655). Mammalian cells were transiently transfected with the corresponding target genes. His-tagged proteins were specifically stained by trisNTA ${ }^{\mathrm{f}}$ with excellent co-localization and signal-to-noise ratio (Pearson's coefficients between 0.90 and 0.96), using confocal laser scanning microscopy (CLSM; Fig. 1b and Supplementary Fig. 1). Strikingly, even at $200 \mathrm{pM}$ of trisNTA ${ }^{\mathrm{f}}$, His-tagged proteins were labelled with high specificity (Supplementary Fig. 2). By analysing a variety of fluorescent dyes, we noticed that trisNTA ${ }^{\text {ATTO565 }}$ targeting produced a higher background compared with tris NTA ATTO647N, tris NTA Alexa647 or trisNTA ${ }^{\text {ATTO655 }}$ (Supplementary Fig. 3). This was assigned to unspecific binding of the ATTO565 dye. Moreover, the superposition of both fluorescence intensity profiles reflects an excellent correlation between the POI expression level and the labelling density of trisNTA ${ }^{\text {Alexa647 }}$ (Fig. 1c,d, Pearson's coefficient $r=0.95)$. Notably, using nanomolar concentrations, tris $\mathrm{NTA}^{\mathrm{f}}$ labelling is significantly more efficient within $30 \mathrm{~min}$ than SNAPf-tag labelling (Supplementary Fig. 4). In contrast, mammalian cells expressing H2B lacking a His tag showed neither tris NTA ${ }^{\mathrm{f}}$ labelling nor unspecific staining (Supplementary Fig. 5). In conclusion, tris $\mathrm{NTA}^{\mathrm{f}}$ targeting at subnanomolar concentrations is highly specific to trace His-tagged proteins. To exploit these benefits further, we combined trisNTA ${ }^{\mathrm{f}}$ with well-established labelling methods for multiplexed protein modification. Specific tris NTA Alexa488 labelling of His10 LaminA (Fig. 1e, green) was successfully achieved in combination with SNAP ${ }^{f}$-tag labelling of H2B (magenta) and antibody labelling of tubulin (red), as well as the lysosomal-associated membrane protein 1 (blue). Thus, the ultra-small interaction pair complements the toolbox of well-established labelling techniques and the nanomolar concentrations perform various avenues in multiplexed labelling.

High-throughput live-cell labelling within mammalian cells. Encouraged by these observations, we aimed at protein labelling in living cells. To transfer tris $\mathrm{NTA}^{\mathrm{f}}$ into cells, we applied microfluidic cell squeezing (Fig. 2a). As the tris $\mathrm{NTA}^{\mathrm{f}}$ probes are chemically diverse relating to the used fluorophores, common transduction strategies are unlikely to efficiently deliver nanomolar concentrations of tris NTA ${ }^{\mathrm{f}}$ into mammalian cells. Specifically, mammalian cells were mechanically pushed ('squeezed') through micrometre constrictions at elevated pressure of $30 \mathrm{psi}$. This approach allows for high cell survival (>90\%) and efficient uptake of tris $\mathrm{NTA}^{\mathrm{f}}$ (up to 80\%; Supplementary Figs 6 and 7). Energy-dependent endocytosis, often observed at cargo transfer with supercharged molecules (Supplementary Fig. 8) or low concentrations of CPPs (Supplementary Fig. 9) ${ }^{17}$, were prevented by performing cell squeezing at $4{ }^{\circ} \mathrm{C}$ (Supplementary Fig. 10).

By squeezing TAP $1^{\text {mVenus-His } 10}$-transfected HeLa cells in the presence of tris $\mathrm{NTA}^{\mathrm{f}}(100 \mathrm{nM})$, we achieved a high-throughput delivery and a high-density labelling, illustrated by an excellent co-localization between both reporter molecules (Fig. 2b). We noticed that both probe delivery by cell squeezing and protein labelling are highly reproducible $(n>20)$. To quantify the 
a
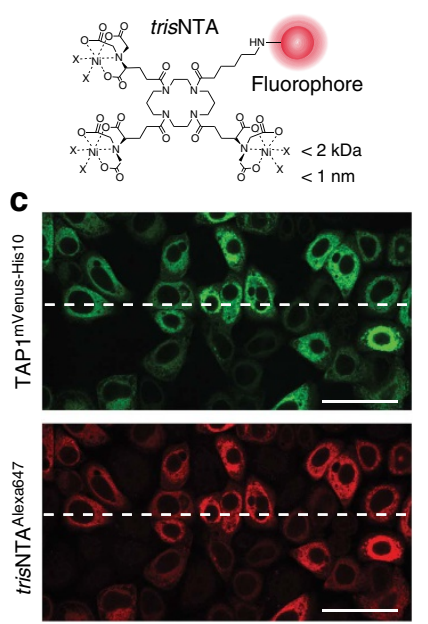

d

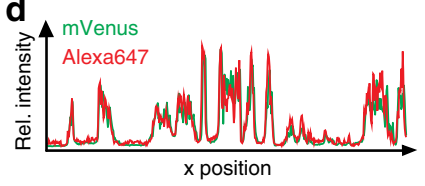

b

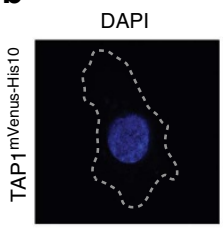

His-tagged $\mathrm{POI}$
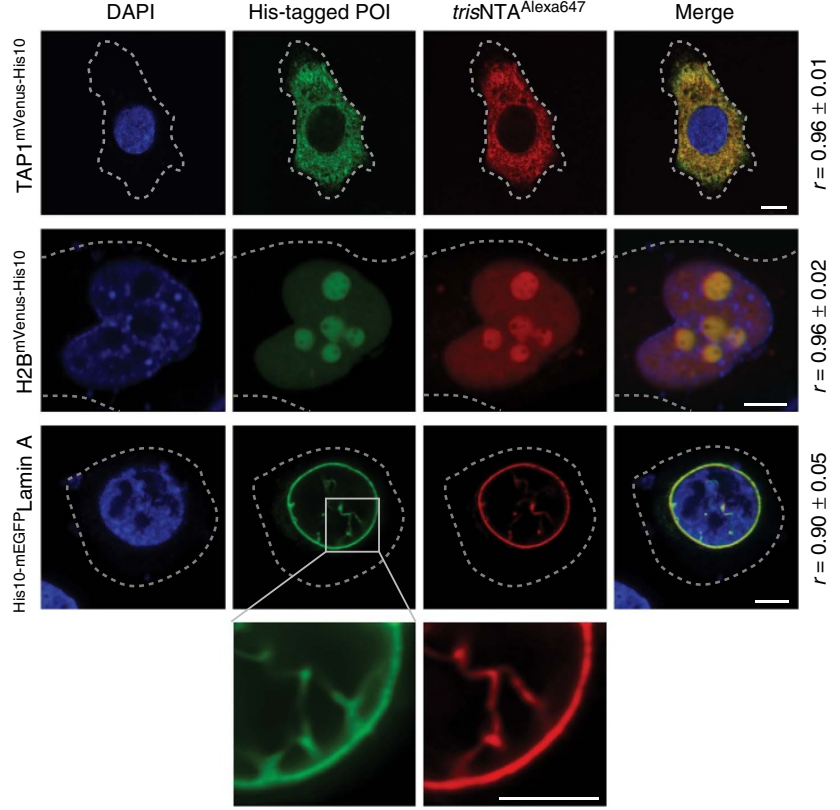

$\mathbf{e}$

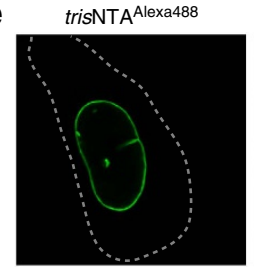

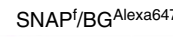

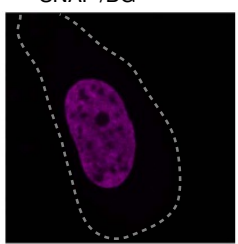

$\alpha$-Tubulin

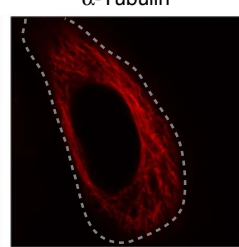

$\alpha$-LAMP1

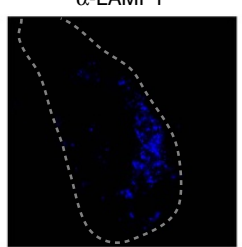

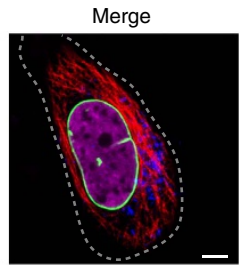

Figure 1 | 'Traceless' tracing of protein assemblies by a minimal lock-and-key recognition pair. (a) Chemical structure of trisNTA conjugated to various organic fluorophores (red circle). (b) Subcellular tracing of His-tagged POIs by trisNTA Alexa647. Cells expressing TAP1 ${ }^{m V e n u s-H i s 10 ~(H e L a ~ K y o t o), ~}$ $\mathrm{H}_{2} \mathrm{~B}^{\mathrm{mV} V e n u s-H i s 10}$ (HeLa) or His10-mEGFP Lamin A (Chinese hamster ovary (CHO-K1)) were fixed and stained with trisNTA Alexa647. Excellent co-localization (merge) between trisNTA Alexa647 (red) and all His-tagged POls (green) was observed. Pearson's coefficients ( $r$ ) were calculated from eight to ten individual images (right). Dashed lines indicate the cell border. (c) Specific labelling of TAP1 ${ }^{\text {mVenus-His10 by trisNTA }}{ }^{\text {Alexa647 }}$ in fixed HeLa Kyoto cells with a Pearson's coefficient of $r=0.95$. (d) Cross-section of relative fluorescence intensities (mVenus and Alexa647), indicated by a horizontal dashed line in c shows excellent correlation of His-tagged POI expression level and trisNTA ${ }^{\text {Alexa647 }}$ staining. (e) Combination of the lock-and-key element with established labelling methods. HeLa Kyoto cells expressing ${ }^{H i s 10}$ LaminA and $\mathrm{H}_{2 \mathrm{~B}}{ }^{\mathrm{SNAPf}}$ were simultaneously labelled with trisNTA Alexa488, benzyl guanine (BG) ${ }^{\mathrm{Alexa} 647}$ and antibodies against tubulin, as well as the lysosomal protein lysosomal-associated membrane protein 1 (LAMP1). Scale bars, $5 \mu \mathrm{m}$ (b,e), $50 \mu \mathrm{m}$ (c).

tris NTA ${ }^{\mathrm{f}}$ delivery, we performed flow cytometry analysis on micromanipulated cells. Thirty-five per cent of transfected cells (54\% of total) were effectively transduced with trisNTA ATTO565 (Fig. 2c), which is $>30 \times$ more efficient for tris $\mathrm{NTA}^{\mathrm{f}}$ delivery than electroporation (Supplementary Fig. 11). Squeezing of up to $1,000,000$ cells per second enables live-cell labelling at high throughput and reproducibility, and hence largely exceeds the efficiency achieved by other direct transfer methods such as microinjection. The massively parallel and constant cell transduction surpasses the stochastic, while variable efficient uptake by CPPs (Supplementary Fig. 9) and is more than 1,000-fold below the micromolar probe concentrations used by elegant self-labelling tags, for example, SNAP $\operatorname{tag}^{3-6}$.

Highly specific protein targeting with minimal disturbance. Analogous micromanipulation and live-cell labelling was applied to $\mathrm{H}_{2} \mathrm{~B}^{\text {mVenus-His10 }}$ - and His10-mEGFP Lamin A-transfected cells (Fig. 2d). Cell transduction was analysed $15 \mathrm{~min}$ and $1 \mathrm{~h}$ after squeezing by CLSM. An excellent co-localization between the tris $\mathrm{NTA}^{\mathrm{f}}$ reporter molecule and the His-tagged POIs was observed (Pearson's coefficients range from 0.81 to 0.93 ), in line with the subcellular localization in chemically arrested cells. Notably, live-cell labelling is independent of cell types, for example, HeLa, HeLa Kyoto, Chinese hamster ovary (CHO-K1) or human embryonic kidney 293 cells (Figs 1 b and 2d). Beyond that, tris NTA ${ }^{\mathrm{f}}$ labelling after removal of the bulky fluorescent protein fully exploited the small size of the lock-and-key element and confirmed again specific labelling in living cells with minimal perturbation (Supplementary Fig. 12). The specificity was validated in cells transfected with $\mathrm{H} 2 \mathrm{~B}^{\mathrm{EGFP}}$ lacking a His tag and untransfected cells (Supplementary Fig. 13). In both cases, neither co-localization of tris NTA ${ }^{\mathrm{f}}$ with $\mathrm{H} 2 \mathrm{~B}^{\mathrm{EGFP}}$ nor unspecific binding was detected. Delivery of nickel-free tris $\mathrm{NTA}^{\mathrm{f}}$ or of free Alexa647 dye showed no labelling in TAP 1 mVenus-His 10 -transfected cells (Supplementary Figs 14 and 15). In contrast, tris NTA Alexa647 (Fig. 2 and Supplementary Fig. 14) and trisNTA ATTO565 (Supplementary Fig. 16) clearly stain TAP1 $1^{\text {mVenus-His10 at }}$ the endoplasmic reticulum membrane after cell squeezing. Noticeably, cell viability of tris $\mathrm{NTA}^{\mathrm{f}}$-transduced cells was negligibly affected 1 and $24 \mathrm{~h}$ after labelling. Similar concentrations of unbound nickel ions inside mammalian cells had no significant toxic effects (Supplementary Fig. 7). In contrast, electroporation entailed more than twofold increased toxicity 
a

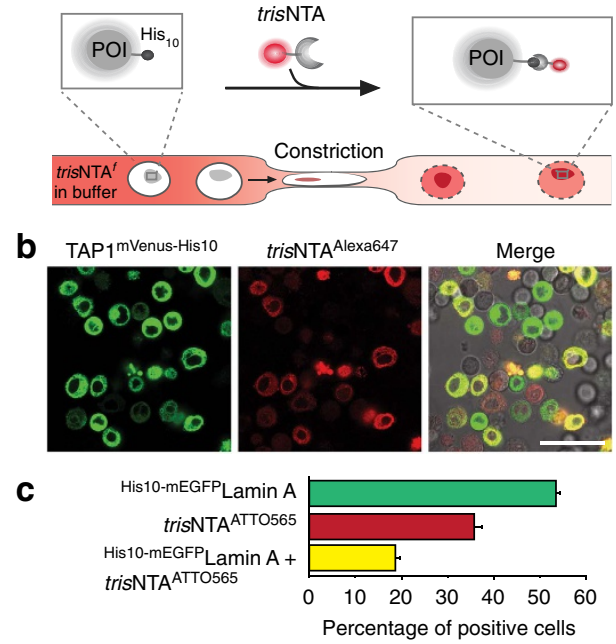

d

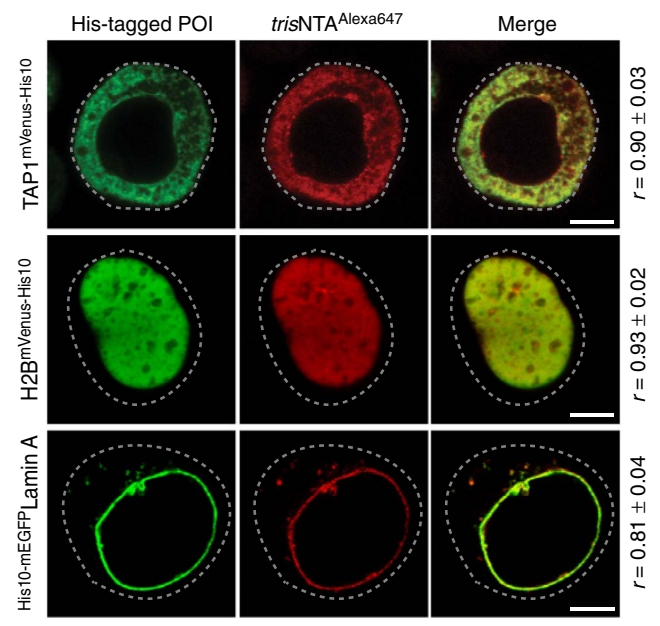

Figure 2 | Live-cell labelling of protein assemblies in distinct subcellular compartments. (a) Delivery of trisNTA into living cells by microfluidic cell squeezing. Cells are pressed through micrometre constrictions of a microfluidic device, to introduce trisNTA ${ }^{f}$ (bottom). Cell passage causes the formation of transient holes in the plasma membrane and enables trisNTA transfer into the cytosol. His-tagged POI (grey) is specifically labelled by fluorophoreconjugated trisNTA (trisNTA ${ }^{f}$, top), resulting in co-localization at the respective subcellular compartment. (b) High-throughput in-cell labelling of TAP1 $^{\text {mVenus-His10 }}$-transfected HeLa cells by trisNTA Alexa647 imaged 10 min after squeezing by CLSM. (c) Flow cytometry analysis of His10-mEGFP ${ }^{\text {Lamin }}$ Atransfected HeLa Kyoto cells, after transduction with $100 \mathrm{nM}$ of trisNTA ATTO565. Fluorescence intensity profiles of mEGFP and ATTO565 revealed that $20 \%$ of all cells were double positive for the His-tagged POI and trisNTA $f$. The error bars indicate the s.d. of three experimental replicates. (d) Specific labelling of His-tagged proteins in living cells at diverse subcellular localizations. trisNTA Alexa647 was delivered to cells and transfected with TAP1 ${ }^{\mathrm{mVenus}-H i s 10}$ (HeLa), His10-mEGFP Lamin A (Chinese hamster ovary (CHO-K1)) or H2B mVenus-His10 (HEK293) by squeezing. High target specificity of trisNTA Alexa647 is represented by excellent co-localization with the POIs (merge). Determined Pearson's coefficients range from 0.81 to 0.93 . Images were taken by CLSM $10 \mathrm{~min}(\mathbf{b})$ or $1 \mathrm{~h}$ (d) after squeezing. Dashed lines indicate the cell border. Scale bars, $50 \mu \mathrm{m}$ (b), $5 \mu \mathrm{m}$ (d).

compared with squeezing (Supplementary Figs 7 and 11). Collectively, nanomolar delivery of tris $\mathrm{NTA}^{\mathrm{f}}$ fully realized the potential of in-cell protein manipulation with minimal perturbation and modification rates exceeding common approaches.

After successful in-cell labelling of different His-tagged proteins, we aimed for in vivo multiplexed labelling by combining tris $\mathrm{NTA}^{\mathrm{f}}$ with well-established labelling methods. By tris NTA ${ }^{\text {Alexa647 delivery via squeezing and subsequent }}$ SNAPf -tag labelling, we achieved specific and distinct targeting of His10LaminA in the presence of two different SNAP ${ }^{f}$-tagged proteins in live cells (Fig. 3a and Supplementary Fig. 17). Hence, tris $\mathrm{NTA}^{\mathrm{f}}$ complements the toolbox for in vivo multiplexed labelling, offering minimal disturbance due to its small size and simultaneously using low nanomolar concentrations.

We next determined the minimal reporter concentration required for specific live-cell labelling. Well-resolved images of TAP $1^{\text {mVenus-His10 }}$ were obtained even at $1 \mathrm{nM}$ of trisNTA Alexa647 (Fig. 3b). Based on previous observations, approximately one-third of the cargo provided during squeezing is the effective intracellular concentration $^{14}$. Thus, the estimated cytosolic concentration of tris $\mathrm{NTA}^{\mathrm{f}}$ further corroborates the high target sensitivity at subnanomolar concentrations $(\sim 300 \mathrm{pM})$. These results are in line with the detection limit of $\sim 200 \mathrm{pM}$ tris NTA ${ }^{\text {Alexa647 }}$ in chemically arrested cells (Supplementary Fig. 2). Hence, this enables the precise adjustment of the effective, intracellular tris NTA ${ }^{\mathrm{f}}$ concentration to improve the signal-to-background ratio, hardly realized by alternative approaches at nanomolar probe concentrations (for example, CPPs, SNAP, CLIP and Halo tag; Supplementary Figs 4 and 9) $3,4,6,18$, and circumvents endocytic uptake observed with supercharged proteins at similar nanomolar concentrations (Supplementary Fig. 8) ${ }^{19}$.

In-cell protein modification with nanometre precision. Incited by this observation, we aimed at temporal and spatial control of protein tracing by light, which depends on low probe concentrations for high signal-to-background ratios. Using photoactivatable tris $\mathrm{NTA}^{\mathrm{f}}$ (PA-tris $\left.\mathrm{NTA}^{\text {ATTO565 }}\right)^{20}$ for dynamic cellular imaging on demand, light-activated in vivo labelling of His10-mEGFP Lamin A was demonstrated up to $24 \mathrm{~h}$ after squeezing (Fig. 3c). Notably, already a 10-s 405-nm light pulse sufficiently activated PA-trisNTA at single-cell level and led to excellent colocalization in a dose-adapted manner. This probe enables in situ labelling at defined time points such as certain mitotic phases and paves the way for live-cell protein tracing with high temporal resolution. The nanomolar concentrations $(\leq 10 \mathrm{nM})$ and in particular the small size of the tag and probe are especially beneficial for advanced microscopy techniques, bringing the fluorophore in 1-nm proximity to the target protein. Hence, we performed live-cell super-resolution microscopy with trisNTA ATTO655 $^{2}$ on His10-mEGFP Lamin A-transfected cells. Using direct stochastic optical reconstruction microscopy $(d S T O R M)^{21}$, Lamin A structures with a high signal-to-noise ratio were obtained in the super-resolved images of live cells. A localization precision of $16.4 \pm 3.1 \mathrm{~nm}$ was achieved, resulting in a resolution of $40 \mathrm{~nm}$ by in-cell tris $\mathrm{NTA}^{\mathrm{ATTO}} 55$ tracing with substantially increased resolution compared with diffractionlimited fluorescence microscopy (Fig. 3d).

\section{Discussion}

We established a high-throughput method for protein labelling inside living cells using a minimalistic lock-and-key probe. Our method is versatile in the choice of the molecular probe, cell type and the subcellular localization of the POIs, a persistent challenge in live-cell analysis. The high-affinity tris NTA/His tag interaction pair enables fast labelling $(\leq 10 \mathrm{~min})$ at subnanomolar concentrations with tunable labelling density and flexibility of cell-impermeable organic fluorophores. Compared with carrier-mediated transport by CPPs ${ }^{11,18}$, delivery of 1,000 -fold 
a
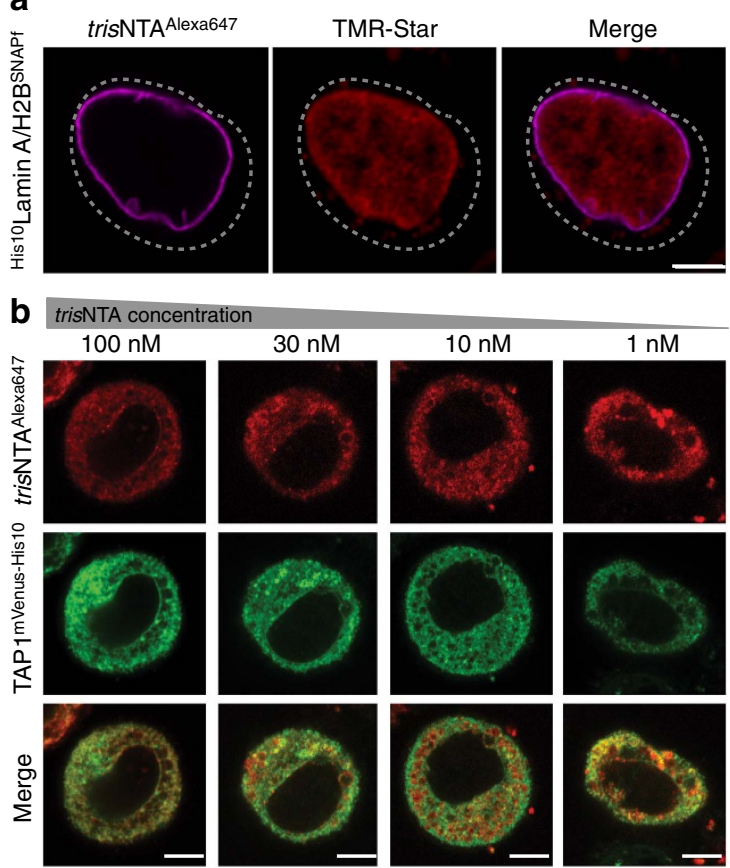

C
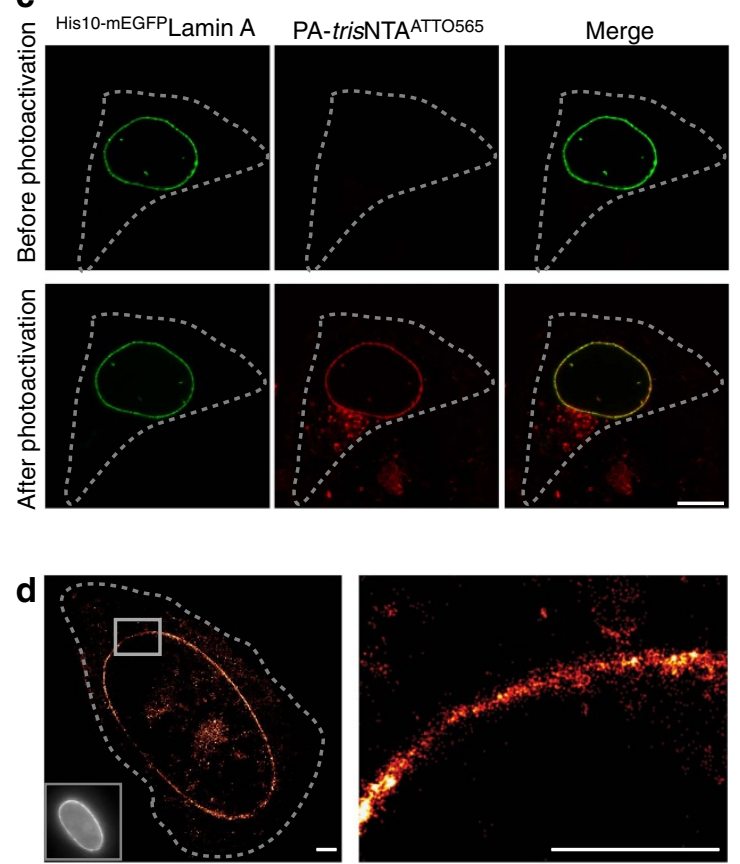

Figure 3 | Light-triggered live-cell labelling and super-resolution microscopy of protein assemblies. (a) Combination of trisNTA labelling with SNAP ${ }^{f}$-tag labelling in living cells. HeLa Kyoto cells co-expressing His10 LaminA and H2B-SNAPf were squeezed in the presence of 100 nM trisNTA Alexa647 (magenta)

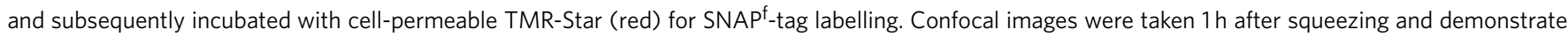
simultaneous specific labelling of the His-tagged LaminA and the SNAPf-tagged H2B. (b) trisNTA Alexa647 concentration scan for tunable labelling of

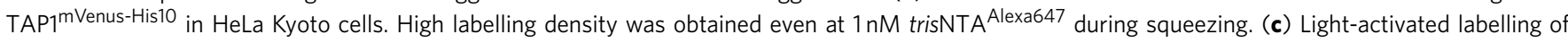
His10-mEGFP Lamin A with PA-trisNTA ATTO565 in living HeLa Kyoto cells. Before photoactivation, no decoration of Lamin A was observed $24 \mathrm{~h}$ after squeezing (top), whereas on illumination fluorescence increase and specific labelling were monitored (bottom). (d) Reconstructed dSTORM image of His10-mEGFP Lamin A labelled with trisNTA ATTO655 (100 nM) in a living HeLa Kyoto cell. Increased spatial resolution ( $\leq 40$ nm) was obtained in live-cell super-resolution imaging of trisNTA ATTO655 by dSTORM (left and magnification right) compared with the wide-field image (left corner, bottom). Images were taken by $\operatorname{CLSM}(\mathbf{a}-\mathbf{c})$ or $\operatorname{dSTORM}(\mathbf{d}) 1 \mathrm{~h}(\mathbf{a}, \mathbf{b}, \mathbf{d})$ or $24 \mathrm{~h}(\mathbf{c})$ after squeezing. Dashed lines indicate the cell border. Scale bars, $5 \mu \mathrm{m}(\mathbf{a}, \mathbf{b}), 10 \mu \mathrm{m}$ (c) and $2 \mu \mathrm{m}$ (d).

lower concentrations ( $\mathrm{nM}$ versus $\mu \mathrm{M}$ ) effectively decreases the fluorescence background. Furthermore, high-throughput analysis with up to 1,000,000 cells per second can be achieved in contrast to microinjection. In addition, tris $\mathrm{NTA}^{\mathrm{f}}$ delivery via squeezing avoids endocytic cargo uptake, frequently observed with low CPP concentrations and supercharged molecules, offering decreased toxicity and a $>30$-fold higher efficiency compared with electroporation. The minimal probe complements the toolbox of well-established labelling techniques such as self-labelling enzymes and can be combined with the latter to achieve distinct labelling of different proteins in fixed as well as in living cells. Moreover, in situ photoactivation of PA-trisNTA allows labelling at defined time points, to trace proteins for dynamic cellular imaging. The achieved close target proximity of the labelling pair substantially improved the localization accuracy in live-cell superresolution microscopy. Remarkable aspects of our approach are the speed, flexibility and efficiency for high-throughput live-cell targeting of proteins even if assembled in stable and transient macromolecular complexes. This study is exploited via one of the smallest high-affinity lock-and-key recognition pairs known so far and allows even multiple cargos to be delivered simultaneously, displaying diverse chemical properties. The quantity of cargo for in-cell manipulation can be precisely tuned and the biological output can in turn be fine-tuned. As the affinity tag is widely used in life sciences and our delivery platform is broadly applicable across cell types, this live-cell labelling method could potentially be implemented across numerous cell-impermeable probes and prodrugs, as well as translated to difficult cell lines including patient-derived cells and embryonic stem cells, providing the opportunity to use these cells for advanced microscopy techniques and live-cell analysis.

\section{Methods}

Plasmid construction. The $\mathrm{H} 2 \mathrm{~B}$ construct $\mathrm{H} 2 \mathrm{~B}^{\text {mVenus-His } 10}$ was generated by consecutive insertion of $\mathrm{H} 2 \mathrm{~B}$ and mVenus-His ${ }_{10}$ into pCDNA3.1 $(+)$ (Life Technologies). mVenus-His ${ }_{10}$ was PCR amplified using Phusion High-Fidelity DNA Polymerase (Fermentas) and the primer pair forward (fw) $5^{\prime}$-GCGCGC GCGGCCGCGTGAGCAAGGGCGAGGAGCTGTTCA-3' (NotI restriction site underlined) and reverse (rev) 5'-GCGCGCTCTAGATTAGTGATGGTGGT GATGATGATG- $3^{\prime}$ (XbaI restriction site underlined), and cloned into the pCDNA3.1 $(+)$ plasmid using the indicated restriction enzymes (Fermentas). Subsequently, H2B was amplified using the primer pair fw $5^{\prime}$-GCGCGCGGTA CCATGCCAGAGCCAGCGAAGTCTGCTCCCGC-3' (Acc65I restriction site underlined, start codon bold) and rev 5'-GCGCGCGCGGCCGCTCTTGG AGCTGGTGTACTTAGTGAC-3' (NotI restriction site underlined), and cloned into the pCDNA3.1 $(+)$ plasmid, amino terminally of mVenus-His ${ }_{10}$. As a template for the amplification of $\mathrm{H} 2 \mathrm{~B}$, the plasmid pEGFP-N1 containing the human H2B sequence (plasmid 11680, Addgene) was used, which also served as control for the specificity of tris $\mathrm{NTA}^{\mathrm{f}}$ for His-tagged POIs in living cells (Supplementary Figs 5 and 13). The plasmid encoding for human Lamin A was generously provided by Dr Sascha Neumann (Institute of Biochemistry, University of Cologne $)^{22}$ and used as template to amplify Lamin A with the primer pair fw $5^{\prime}$-GCGCGCCTCGAGCTATGGAGACCCCGTCCCAGCGGCGCGCCACCC G-3' (XhoI restriction site underlined) and rev 5'-GCGCGCGATATCTTACA TGATGCTGCAGTTCTGGGGGCTCTGGG-3' (EcoRV restriction site underlined, stop codon bold). Lamin A was inserted into the pcDXC3GMS plasmid (Addgene) already containing His 10 -mEGFP, leading to the fusion gene encoding for His10-mEGFP Lamin A. To generate the plasmid containing His10 Lamin A without a fluorescent protein, Lamin A was amplified via PCR, simultaneously introducing a $\mathrm{His}_{10}$-tag with the primer pair fw $5^{\prime}$-GCGCGCGGATCCACCATGC ACCATCATCATCATCATCACCACCATCACTCCGGACTCAGATCTCGAGT 
CATGGAGACCC-3' (BamHI restriction site underlined, start codon bold, His $_{10}$-tag italic) and rev $5^{\prime}$-GCGCGCGCGGCCGCTTACATGATGCTGC AGTTCTGGGGGCTCTGGG-3' (NotI restriction site underlined, stop codon bold). Indicated restriction sites were used to insert this PCR product into the pCDNA3.1 $(+)$ vector. The pSNAPf ${ }^{\mathrm{f}}$-H2B and pSNAP ${ }^{\mathrm{f}}$-Cox8A plasmids (New England Biolabs) were used for SNAPf-tag labelling. In addition, a plasmid coding for the core domain of TAP1, tagged with mVenus-His ${ }_{10}$ $\left(\mathrm{TAP}^{\mathrm{mVenus}-\mathrm{His} 10}\right)$ was used as previously described ${ }^{23}$.

Cell culture and transfection. HeLa cells, HeLa Kyoto cells, Chinese hamster ovary (CHO-K1) cells and human embryonic kidney 293 cells were maintained in DMEM medium with $4.5 \mathrm{gl}^{-1}$ glucose (Gibco), supplied with $10 \%$ (v/v) FCS (Gibco) in T75 cell culture flasks (Greiner). Every 2-3 days, cells were passaged using PBS (Sigma-Aldrich) and 0.5\% trypsin/0.02\% EDTA/PBS (GE Healthcare). All cell lines were cultivated in a humidified tissue culture incubator at $37{ }^{\circ} \mathrm{C}$ and $5 \% \mathrm{CO}_{2}$. Mycoplasma contamination tests were carried out regularly, following the guidelines described ${ }^{24}$. Transient transfection was performed with Lipofectamine 2000 (Life Technologies), following the manufacturer's instructions. For fixation and staining, $2 \times 10^{4}$ cells per well were seeded into eight wells on cover glass II slides (Sarstedt) and transfected with $0.2 \mu \mathrm{g}$ DNA per well. For squeezing experiments, $8 \times 10^{5}$ cells were seeded into six-well cell culture plates (Greiner) and transfected with $2 \mu \mathrm{g}$ DNA per well. After transfection, cells were incubated $12-48 \mathrm{~h}$ at $37^{\circ} \mathrm{C}$ and $5 \% \mathrm{CO}_{2}$ until experiments were performed.

Cell viability test. To analyse cell viability after squeezing, the Sytox Blue Dead Cell stain (Life Technologies) was used to stain cells with a permeable plasma membrane. Cells were squeezed in the presence of $100 \mathrm{nM}$ trisNTA ${ }^{\text {Alexa647 }}, 100 \mathrm{nM}$ Alexa647 dye or $500 \mathrm{nM} \mathrm{NiCl}_{2}$, followed by incubation with $1 \mu \mathrm{M}$ of Sytox Blue Dead Cell Stain (20 min, room temperature (RT)) at different time points. Cell viability analysis was performed using the Attune flow cytometer (Life Technologies) and data were processed using FlowJo 7.6.5 (Tree Star Inc.). Before detaching the cells, the supernatant was collected to avoid altering the results by removing dead cells during washing. Identical Sytox Blue Dead Cell Staining was conducted with cells after electroporation (described above), followed by flow cytometry analysis of cell viability and uptake of tris $\mathrm{NTA}^{\text {ATTO565. All }}$ experiments were performed in triplicates and error bars indicate the s.d.

Confocal imaging. Imaging was performed using the confocal laser scanning TCS SP5 microscope (Leica) and a Plan-Appochromat $63 \times 1.4$ Oil differential interference contrast objective. Images were acquired sequentially to avoid cross-talk. The following laser lines were used for excitation: $405 \mathrm{~nm}$ (diode laser) for 4,6-diamidino-2-phenylindole, Hoechst and Sytox Blue Dead Cell Stain; $488 \mathrm{~nm}$ (argon laser) for monomeric enhanced green fluorescent protein (mEGFP), Alexa488, MitoTracker Green and Syto16; 488 or $514 \mathrm{~nm}$ (argon laser) for mVenus; $565 \mathrm{~nm}$ (diode-pumped solid-state laser) for ATTO565 and TMR-Star; and $633 \mathrm{~nm}$ (helium-neon laser) for ATTO647N, Alexa647 and ATTO655. For image analysis,

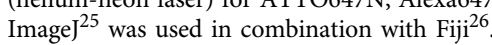

Super-resolution imaging. A custom-built microscope was used for super-resolution imaging of tris NTA ${ }^{\text {ATTO655-labelled His10-mEGFP }}{ }^{\text {Lamin }} \mathrm{A}^{27}$. Samples were illuminated with $488 \mathrm{~nm}$ (Sapphire $488 \mathrm{LP}$, Coherent) and $643 \mathrm{~nm}$ (iBeam smart, Toptica Photonics) laser beams in total internal reflection fluorescence mode. The excitation light was focused on the back focal plane of a $100 \times$ oil objective (PLAPO $100 \times$, total internal reflection fluorescence mode, numerical aperture 1.45 , Olympus) mounted on an inverted microscope (Olympus IX71). The emission was recorded using an electron multiplying charge-coupled device camera (Ixon3, Andor) with frame-transfer mode, $5.1 \times$ pre-amplifier gain and electron multiplying (EM) gain set to 200. For every sample, 40,000 images were recorded at a frame rate of $33 \mathrm{~Hz}$ and image reconstruction was performed with rapidSTORM ${ }^{28}$. The localization $\left(\sigma_{\text {loc }}\right)$ precision of the $d$ STORM images was calculated to $16.4 \pm 3.1 \mathrm{~nm}$ and a resolution of $\leq 40 \mathrm{~nm}$ (for $d S T O R M$ image see Fig. 3d). Calculations were performed according to Mortensen et al. ${ }^{29}$

Fixation and trisNTA ${ }^{f}$ labelling. Before fixation, cells were washed with PBS (Sigma-Aldrich). Fixation with $4 \%$ formaldehyde (Roth)/PBS for $15 \mathrm{~min}$ at RT was followed by quenching using $50 \mathrm{mM}$ glycine/PBS (10 min, RT; Roth) and permeabilization with $0.1 \%$ Triton X-100/PBS (10 min, RT; Roth). After blocking with 5\% (w/v) BSA (Albumin Fraction V, Roth) in PBS (1 h, RT), cells were stained with $100 \mathrm{nM}$ of tris $\mathrm{NTA}^{\mathrm{f}}$ in $1 \%(\mathrm{w} / \mathrm{v})$ BSA/PBS. Cells were stained with $0.1 \mu \mathrm{g} \mathrm{ml}^{-1} 4^{\prime}, 6$-diamidino-2-phenylindole (Sigma-Aldrich) in 1\% BSA/PBS for 30 min- $1 \mathrm{~h}$ at RT. After washing with $5 \%$ BSA/PBS $(3 \times)$, cells were postfixed with $2 \%$ formaldehyde/PBS ( $15 \mathrm{~min}, \mathrm{RT}$ ) and stored in PBS until confocal imaging was performed. When tris NTA ${ }^{\mathrm{f}}$ labelling was combined with antibody labelling, $100 \mathrm{nM}$ trisNTA was applied together with the primary antibodies for $1 \mathrm{~h}$ at RT. After subsequent washing with PBS, cells were incubated with secondary antibodies. Antibodies were all diluted in 1\% BSA/PBS as follows: rabbit $\alpha$-tubulin (Abcam, 1:350), mouse $\alpha$-human CD107a (LAMP-1; Biolegend, 1:1,000), goat $\alpha$-rabbit ${ }^{\mathrm{Cy} 3}$ (Abcam, 1:1,000) and goat $\alpha$-mouse ${ }^{\text {PacificBlue }}$ (Life Technologies,
1:1,000). tris NTA was conjugated with the corresponding fluorescent dyes by reacting amino-trisNTA with the N-hydroxysuccinimide (NHS)-activated dye, respectively. After RP- $\mathrm{C}_{18}$ HPLC chromatography, the multivalent chelator head was loaded with $\mathrm{Ni}^{2}+\left(10 \mathrm{mM} \mathrm{NiCl}_{2}\right.$ in $20 \mathrm{mM}$ HEPES, pH 7.0) and purified by anion exchange chromatography (HiTrap Q HP; GE Healthcare) ${ }^{11}$.

SNAPf-tag labelling in fixed cells. HeLa Kyoto cells transfected with $\mathrm{H}_{2} \mathrm{~B}^{\mathrm{SNAPf}}$ were labelled with O6-benzyl guanine $e^{\text {Alexa647 }}$ (New England Biolabs) $24 \mathrm{~h}$ after transfection. After washing with PBS, cells were fixed with $4 \%$ formaldehyde/PBS (10 min, RT), washed twice with PBS and subsequently permeabilized using $0.1 \%$ Triton X-100/PBS (10 min, RT). Cells were washed three times with $0.5 \%$ BSA/PBS and incubated with 10 or $100 \mathrm{nM}$ of benzyl guanine $\mathrm{Alexa}^{\mathrm{N}} 47$ for $30 \mathrm{~min}$ at $37^{\circ} \mathrm{C}$. After three washing steps with $0.1 \%$ Triton X-100/0.5\% BSA/PBS and two washing steps with PBS, imaging was conducted by CLSM. In case of combined trisNTA ${ }^{\mathrm{f}}$ and SNAP ${ }^{\mathrm{f}}$-tag labelling, SNAPf -tag labelling was performed first, followed by tris $\mathrm{NTA}^{\mathrm{f}}$ labelling as described above.

trisNTA ${ }^{\mathbf{f}}$ delivery via cell squeezing. Squeezing was performed using a chip with constrictions of $7 \mu \mathrm{m}$ in diameter and $10 \mu \mathrm{m}$ in length (CellSqueeze 10-(7)x1, SQZbiotech), if not otherwise stated. In all microfluidic experiments, a cell density of $1.5 \times 10^{6}$ cells per $\mathrm{ml}$ in $10 \%(\mathrm{v} / \mathrm{v})$ FCS/PBS were squeezed through the chip at a pressure of $30 \mathrm{psi}$. Transduction was conducted at $4{ }^{\circ} \mathrm{C}$, to block cargo uptake by endocytosis. During squeezing, the following cargo concentrations were used: $0.1-100 \mathrm{nM}$ of $\operatorname{trisNTA}^{\mathrm{f}}(\mathrm{f}=$ ATTO565, ATTO647N, Alexa647 or ATTO655), $200 \mathrm{nM}$ of PA-trisNTA ${ }^{\text {ATTO565 }}$ and $100 \mathrm{nM}$ of free Alexa647 (Molecular Probes). After squeezing, cells were incubated for $5 \mathrm{~min}$ at $4{ }^{\circ} \mathrm{C}$, to reseal the plasma membrane ${ }^{15}$. Squeezed cells were washed with DMEM containing $10 \%$ FCS and $10 \mathrm{mM}$ histidine (Sigma-Aldrich), to remove unspecifically bound trisNTA ${ }^{\mathrm{f}}$ from the cell surface, seeded into eight wells on cover glass II slides (Sarstedt) in DMEM containing $10 \% \mathrm{FCS}$ and cultured at $37^{\circ} \mathrm{C}$ and $5 \% \mathrm{CO}_{2}$. Confocal imaging was performed at different time points $(0.25,0.5,1,2$ and $24 \mathrm{~h})$ after squeezing. As a control for endosomal uptake, cells were incubated with $100 \mathrm{nM}$ of trisNTA ${ }^{\mathrm{f}}$ at RT and $4{ }^{\circ} \mathrm{C}$ without microfluidic cell manipulation (Supplementary Fig. 10). In case of combined tris $\mathrm{NTA}^{\mathrm{f}}$ and SNAPf ${ }^{\mathrm{f}}$-tag labelling, cells were first squeezed in the presence of $100 \mathrm{nM}$ trisNTA ${ }^{\text {Alexa647 }}$ and $3 \mu \mathrm{M}$ SNAP-Cell TMR-Star (New England Biolabs) was added $5 \mathrm{~min}$ after squeezing. After $15 \mathrm{~min}$ incubation at $37^{\circ} \mathrm{C}$ and $5 \%$ $\mathrm{CO}_{2}$, cells were washed with $10 \%$ FCS/DMEM and incubated again 30 min under standard cell culture conditions before CLSM imaging was performed. To visualize mitochondria in case of Cox8 $\mathrm{A}^{\text {SNAPf }}$ labelling MitoTracker ${ }^{\text {Alexa488 }}$ was added 10 min prior imaging.

trisNTA $^{\mathbf{f}}$ delivery by the CPP Tat $_{\mathbf{4 9 - 5 7}}$. TAP ${ }^{\text {mVenus-His10 }}$-transfected HeLa Kyoto cells were incubated with different concentrations $(10 \mu \mathrm{M}$ and 100,10 and $1 \mathrm{nM})$ of a non-covalent complex composed of Tat $4_{49-57}^{\text {iis }}$ and trisNTA ${ }^{\text {Alexa647 }}$ for 30 min. After washing with $10 \% \mathrm{FCS} / \mathrm{DMEM}$ and $\mathrm{PBS}$ at $37^{\circ} \mathrm{C}$, trisNTA uptake and labelling of $\mathrm{His}_{10}$-tagged TAP was analysed by live-cell imaging via CLSM ${ }^{11}$.

trisNTA ${ }^{\mathbf{f}}$ delivery via supercharged GFP $\left(\right.$ GFP $\left.^{36+}\right)$. trisNTA $^{\text {ATTO565 }}(100 \mathrm{nM})$ and ${ }^{\mathrm{His} 6} \mathrm{GFP}^{36}+(100 \mathrm{nM})$ were incubated for $30 \mathrm{~min}$ at RT to form the trisNTAHis tag complex. HeLa cells were washed with PBS and treated with the pre-formed complex at $37^{\circ} \mathrm{C}$. In vivo uptake was immediately followed by CLSM. After $20 \mathrm{~min}$, cells were washed three times with PBS and $20 \mathrm{U} \mathrm{ml}^{-1}$ heparin/PBS $(2 \times)$, to remove the complex from the plasma membrane. Internalization of trisNTA/ ${ }_{\text {His }} \mathrm{GFP}^{36+}$ was analysed by CLSM after washing three times with PBS. ${ }^{H i s 6} \mathrm{GFP}^{36+}$ was expressed in BL21(DE3) Escherichia coli. After lysis by sonication in $2 \mathrm{M} \mathrm{NaCl} / \mathrm{PBS}$, ${ }^{\mathrm{His} 6} \mathrm{GFP}^{36+}$ proteins were purified via immobilized metal ion affinity chromatography using Ni Sepharose 6 Fast Flow (GE Healthcare). Elusion was performed with $500 \mathrm{mM}$ imidazole before desalting of the eluted protein was conducted with PD-10 desalting columns (GE Healthcare) ${ }^{19}$.

trisNTA $^{\mathbf{f}}$ delivery via electroporation. Trypsinized HeLa Kyoto cells were per-

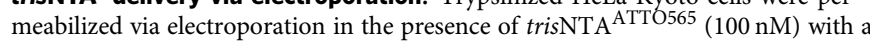
Nucleofector Device (Lonza) using Nucleofector Kit V and program I-013. Transduced cells were washed with 10\% FCS/DMEM and plated into eight-well cover glass II slides. To quantify trisNTA ${ }^{\mathrm{f}}$ uptake, flow cytometry analysis (as described above) and CLSM imaging was performed $1 \mathrm{~h}$ after electroporation Before CLSM imaging, transduced HeLa cells were incubated with Sytox Blue Dead Cell Stain or Syto16 (Life Technologies) live cell stain, to distinguish between dead and live cells. Cell viability was analysed by flow cytometry using Sytox Blue Dead Cell Stain (see above).

Photoactivation of PA-trisNTA $\mathbf{f}^{\mathrm{f}}$. HeLa Kyoto cells, transfected with His10-mEGFP Lamin A, were squeezed in the presence of PA-trisNTA ATTO565 $(200 \mathrm{nM})^{20}$ and incubated at $37{ }^{\circ} \mathrm{C}$ and $5 \% \mathrm{CO}_{2}$. Imaging via CLSM and photoactivation was conducted 2 or $24 \mathrm{~h}$ after squeezing, after cells reattached to the glass surface. PAtrisNTA ${ }^{\text {ATTO565 }}$ was photoactivated in single cells by illumination with the $405-\mathrm{nm}$ 
laser for $10 \mathrm{~s}$. Imaging of both channels (mEGFP and ATTO565) was performed before and directly after photoactivation.

\section{References}

1. Jones, S. A., Shim, S. H., He, J. \& Zhuang, X. Fast, three-dimensional superresolution imaging of live cells. Nat. Methods 8, 499-508 (2011).

2. Wombacher, R. et al. Live-cell super-resolution imaging with trimethoprim conjugates. Nat. Methods 7, 717-719 (2010).

3. Gautier, A. et al. An engineered protein tag for multiprotein labeling in living cells. Chem. Biol. 15, 128-136 (2008).

4. Keppler, A. et al. A general method for the covalent labeling of fusion proteins with small molecules in vivo. Nat. Biotechnol. 21, 86-89 (2003).

5. Los, G. V. et al. HaloTag: a novel protein labeling technology for cell imaging and protein analysis. ACS Chem. Biol. 3, 373-382 (2008).

6. O'Hare, H. M., Johnsson, K. \& Gautier, A. Chemical probes shed light on protein function. Curr. Opin. Struct. Biol. 17, 488-494 (2007).

7. Uttamapinant, C. et al. A fluorophore ligase for site-specific protein labeling inside living cells. Proc. Natl Acad. Sci. USA 107, 10914-10919 (2010).

8. Zhou, Z. et al. Genetically encoded short peptide tags for orthogonal protein labeling by sfp and AcpS phosphopantetheinyl transferases. ACS Chem. Biol. 2, 337-346 (2007).

9. Griffin, B. A., Adams, S. R. \& Tsien, R. Y. Specific covalent labeling of recombinant protein molecules inside live cells. Science 281, 269-272 (1998)

10. Lata, S., Gavutis, M., Tampé, R. \& Piehler, J. Specific and stable fluorescence labeling of histidine-tagged proteins for dissecting multi-protein complex formation. J. Am. Chem. Soc. 128, 2365-2372 (2006).

11. Wieneke, R. et al. Live-cell targeting of His-tagged proteins by multivalent N-nitrilotriacetic acid carrier complexes. J. Am. Chem. Soc. 136, 13975-13978 (2014).

12. Giannone, G. et al. Dynamic superresolution imaging of endogenous proteins on living cells at ultra-high density. Biophys. J. 99, 1303-1310 (2010).

13. Wieneke, R., Raulf, A., Kollmannsperger, A., Heilemann, M. \& Tampé, R. SLAP: small labeling pair for single-molecule super-resolution imaging. Angew. Chem. Int. Ed. Engl. 54, 10216-10219 (2015).

14. Sharei, A. et al. A vector-free microfluidic platform for intracellular delivery. Proc. Natl Acad. Sci. USA 110, 2082-2087 (2013).

15. Sharei, A. et al. Plasma membrane recovery kinetics of a microfluidic intracellular delivery platform. Integr. Biol. (Camb) 6, 470-475 (2014)

16. Mayerhofer, P. U. \& Tampé, R. Antigen translocation machineries in adaptive immunity and viral immune evasion. J. Mol. Biol. 427, 1102-1118 (2014).

17. Brock, R. The uptake of arginine-rich cell-penetrating peptides: putting the puzzle together. Bioconjug. Chem. 25, 863-868 (2014).

18. Vives, E., Brodin, P. \& Lebleu, B. A truncated HIV-1 Tat protein basic domain rapidly translocates through the plasma membrane and accumulates in the cell nucleus. J. Biol. Chem. 272, 16010-16017 (1997).

19. McNaughton, B. R., Cronican, J. J., Thompson, D. B. \& Liu, D. R. Mammalian cell penetration, siRNA transfection, and DNA transfection by supercharged proteins. Proc. Natl Acad. Sci. USA 106, 6111-6116 (2009).

20. Laboria, N., Wieneke, R. \& Tampé, R. Control of nanomolar interaction and in situ assembly of proteins in four dimensions by light. Angew. Chem. Int. Ed. Engl. 52, 848-853 (2013).

21. Heilemann, M. et al. Subdiffraction-resolution fluorescence imaging with conventional fluorescent probes. Angew. Chem. Int. Ed. Engl. 47, 6172-6176 (2008).

22. Yang, L. et al. Mutations in LMNA modulate the lamin A--Nesprin-2 interaction and cause LINC complex alterations. PLoS ONE 8, e71850 (2013).
23. Parcej, D., Guntrum, R., Schmidt, S., Hinz, A. \& Tampé, R. Multicolour fluorescence-detection size-exclusion chromatography for structural genomics of membrane multiprotein complexes. PLoS ONE 8, e67112 (2013).

24. Uphoff, C. C. \& Drexler, H. G. Detection of Mycoplasma Contamination in Cell Cultures. Curr. Protoc. Mol. Biol. 106, 28.4:28.4.1-28.4.14 (2014).

25. Girish, V. \& Vijayalakshmi, A. Affordable image analysis using NIH Image/ ImageJ. Indian J. Cancer 41, 47 (2004).

26. Schindelin, J. et al. Fiji: an open-source platform for biological-image analysis Nat. Methods 9, 676-682 (2012).

27. Dietz, M. S., Fricke, F., Kruger, C. L., Niemann, H. H. \& Heilemann, M. Receptor-ligand interactions: binding affinities studied by single-molecule and super-resolution microscopy on intact cells. Chemphyschem 15, 671-676 (2014).

28. Wolter, S. et al. Real-time computation of subdiffraction-resolution fluorescence images. J. Microsc. 237, 12-22 (2010).

29. Mortensen, K. I., Churchman, L. S., Spudich, J. A. \& Flyvbjerg, H. Optimized localization analysis for single-molecule tracking and super-resolution microscopy. Nat. Methods 7, 377-381 (2010)

\section{Acknowledgements}

The German Research Foundation (Cluster of Excellence-Macromolecular Complexes to R.W., M.H. and R.T., as well as CRC 807, SPP 1623 and RTG 1986 to R.T. and SFB 807 to M.H.) supported the work. We thank Drs Sascha Neumann (Institute of Biochemistry, University of Cologne, Germany) and Ulrich Rothbauer (The Natural and Medical Sciences Institute, University of Tübingen, Germany) for generously providing us with the original Lamin A construct and the HeLa Kyoto cells, respectively. Furthermore, we thank Valentina Herbring and Dr Peter Mayerhofer for help with flow cytometry, and Markus Braner for helpful suggestions on the manuscript.

\section{Author contributions}

A.K. designed and performed the cell squeezing and labelling experiments. A.S. determined the squeezing efficiency. A.S., R.L. and K.F.J. designed and provided the microfluidic devices. A.R. and M.H. performed the dSTORM imaging and analysis. A.K., R.W. and R.T. wrote the manuscript and analysed the data. R.W. and R.T. conceived the ideas and directed the work.

\section{Additional information}

Supplementary Information accompanies this paper at http://www.nature.com/ naturecommunications

Competing financial interests: The authors declare no competing financial interests.

Reprints and permission information is available online at http://npg.nature.com/ reprintsandpermissions/

How to cite this article: Kollmannsperger, A. et al. Live-cell protein labelling with nanometre precision by cell squeezing. Nat. Commun. 7:10372 doi: $10.1038 /$ ncomms10372 (2016).

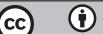

This work is licensed under a Creative Commons Attribution 4.0 International License. The images or other third party material in this article are included in the article's Creative Commons license, unless indicated otherwise in the credit line; if the material is not included under the Creative Commons license, users will need to obtain permission from the license holder to reproduce the material. To view a copy of this license, visit http://creativecommons.org/licenses/by/4.0/ 